\title{
Linking Geometric Mass Hierarchy with Threefold Family Replication
}

\author{
Aharon Davidson and Tomer Schwartz \\ Physics Department, Ben-Gurion University of the Negev, \\ Beer-Sheva 84105, Israel \\ (davidson@bgumail.bgu.ac.il) \\ Raymond R. Volkas \\ School of Physics, Research Centre for High Energy Physics, \\ The University of Melbourne, Parkville 3052, Australia \\ (r.volkas@physics.unimelb.edu.au)
}

\begin{abstract}
A link is established between the observed (approximate) geometric mass hierarchy of quarks and leptons and the triangular structure of their tenable flavor representations. This singles out $S U(3)_{H}$ as the horizontal flavor group, thereby linking the Fermi mass hierarchy with the threefold family replication. These linkages are exploited within a flavor-chiral $S U(3)_{H}$ model, with fermions and Higgs bosons in the $\underline{3} \oplus \underline{6}^{*}$ representation. The model is Left-Right symmetric and utilizes the universal see-saw mechanism with a geometric mass suppression pattern. Given certain assumptions, the model produces the following mass-ratio (rather than square-mass-ratio) mixing angle relations $V_{c b}=-V_{t s}=\frac{m_{s}}{m_{b}}+\frac{m_{c}}{m_{t}}, V_{u b}=\left(2 \frac{m_{c}}{m_{t}}-\frac{m_{s}}{m_{b}}\right) \theta_{C}, V_{t d}=\left(2 \frac{m_{s}}{m_{b}}-\frac{m_{c}}{m_{t}}\right) \theta_{C}$, and fixes the $\frac{m_{u}}{m_{d}}$ ratio.

PACS numbers: 11.30.Hv, 12.10.Kt, 12.15.Ff, 12.15.Hh, 12.60.-i
\end{abstract}

\section{INTRODUCTION}

Many ideas have been proposed as to how the pattern of quark and lepton masses and mixings observed in nature might be better understood. These ideas include horizontal symmetry [1,2], partial [3] and full [4] grand unification, radiative mass generation [5], and the universal see-saw [6] mechanism. The latter is a simple Dirac-type extension of the see-saw mechanism [7] invented for Majorana neutrinos. While all of these ideas are very interesting, it is fair to say that no uniquely compelling model has yet emerged which provides both a qualitative and a quantitative resolution of the fermion mass problem within an elegant theoretical framework.

In this paper, we present a theoretical structure for quark masses and mixings which provides for a qualitative understanding of the Fermi mass hierarchy, and which suggests five phenomenologically successful quantitative mass and mixing angle relations. Our theory is based on an intricate marriage between horizontal flavorchiral $S U(3)_{H}$ symmetry and the universal see-saw mechanism. This non-trivial amalgam exploits the ability of the universal see-saw to explain why most quark masses are much lighter than the electroweak scale together with the ability of spontaneously broken $S U(3)_{H}$ to discriminate among families. It thus utilizes the best features of universal see-saw and horizontal symmetry while avoiding their well-known inadequacies when used in isolation. The core of the model is a strong linkage between the observed (approximate) geometric mass hierarchy of quarks and leptons and the specific horizontal symmetry group $S U(3)_{H}$. This symmetry group, in turn, is strongly linked to threefold family replication.

We first discuss the three important hints that are pro- vided by the combination of mass hierarchy phenomenology and theoretical ideas: (i) the Left-Right symmetric universal see-saw mechanism, (ii) $p$-fold universal see-saw suppression pointing towards $S U(2)_{H}$ horizontal symmetry, and (iii) the geometric mass hierarchy and its intimate relation with $S U(3)_{H}$ horizontal symmetry. After discussing these hints in Section II, we present in Section III the theoretical structure that combines all of these ideas and resolves several apparent incompatibilities among them. The theory is then used to extract phenomenologically successful mass and mixing relations for quarks. We summarize our results in Section IV and discuss some important open problems.

\section{HINTS TOWARDS A THEORY OF FLAVOR}

\section{Hint 1: Left-Right Symmetric Universal See-saw Mechanism}

Consider the Left-Right symmetric electroweak gauge group $G_{L R}=S U(2)_{L} \otimes S U(2)_{R} \otimes U(1)_{B-L}$, and focus, say, on the quark sector. In addition to the standard left-handed and right-handed quarks

$$
\begin{aligned}
q_{L} & \sim(\underline{2}, \underline{1})_{1 / 3}, \\
q_{R} & \sim(\underline{1}, \underline{2})_{1 / 3},
\end{aligned}
$$

introduce the exotic vector-like quarks

$$
\begin{aligned}
Q_{L} & \sim(\underline{1}, \underline{1})_{4 / 3,-2 / 3}, \\
Q_{R} & \sim(\underline{1}, \underline{1})_{4 / 3,-2 / 3},
\end{aligned}
$$

with matching electric charges. The structure of the prototype mass matrix is then 


$$
\left.\begin{array}{ccc} 
& q_{R} & Q_{R} \\
q_{L} & (\underline{2}, \underline{2})_{0} & (\underline{2}, \underline{1})_{ \pm 1} \\
\hline Q_{L} & (\underline{1}, \underline{2})_{ \pm 1} & (\underline{1}, \underline{1})_{0}
\end{array}\right),
$$

with the various entries denoting the electroweak assignment of the corresponding Higgs multiplets and/or bare mass terms. It acquires the universal see-saw form

$$
\left(\begin{array}{cc}
0 & \ell \\
r & M
\end{array}\right)
$$

provided that (i) the putative $(\underline{2}, \underline{2})_{0}$ Higgs multiplet is absent, and (ii) the symmetry breaking chain is governed by

$$
\ell \ll r \ll M,
$$

where $\ell=\left\langle(\underline{1}, \underline{2})_{ \pm 1}\right\rangle, r=\left\langle(\underline{2}, \underline{1})_{ \pm 1}\right\rangle$, and $M$ stands for the $(\underline{1}, \underline{1})_{0}$ bare mass term. The eigenmasses are approximately given by

$$
\begin{aligned}
& m_{\text {light }} \simeq \frac{r}{M} \ell, \\
& m_{\text {heavy }} \simeq M .
\end{aligned}
$$

Compared with the electroweak scale $\ell$, the light eigenmass is suppressed by the universal see-saw hierarchy parameter $\epsilon \equiv \frac{r}{M}$. The corresponding light eigenstate, given approximately by $q-\frac{r}{M} Q$, exhibits close to standard electroweak interactions. Once heavy vector-like leptons are also introduced into the scheme, the see-saw suppression becomes universal. This universality comes with a bonus. Namely, the Dirac-mass see-saw suppression in the charged lepton sector automatically produces [6] the Gell-Mann-Yanagida see-saw suppression in the Majorana-neutrino sector.

Although the naive universal see-saw is unable to account for the full complexity of the observed fermion mass spectrum, it is possible to extend the idea to incorporate both unsuppressed and singly-suppressed eigenmasses

$$
\begin{aligned}
& m_{1} \sim \ell, \\
& m_{2} \sim \ell \epsilon .
\end{aligned}
$$

This can be achieved through a toy two-family model [8] where Left-Right symmetry is accompanied by a horizontal $U(1)_{H}$ flavor group. In this model, the full mass matrix has dimension $1+2+1=4$. Its building blocks (produced when Cabibbo mixing is switched off) form the Left-Right symmetric sequence

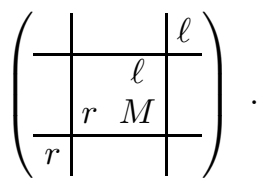

Note that the unsuppressed eigenmass $\sim \ell$ has, through Left-Right symmetry, a medium-heavy partner $\sim r$. The main problem is that such a toy model, while respecting Left-Right symmetry, cannot be easily modified to also incorporate a doubly-suppressed eigenmass $\sim \ell \epsilon^{2}$.

\section{Hint 2: From $p$-fold Suppression to $S U(2)_{H}$}

Motivated by the highly suppressed up and down quark masses, we now attempt, as an abstract exercise, to generalize the universal see-saw matrix to produce a $p$-fold suppressed eigenvalue $\sim \ell \epsilon^{p}$. The prescription is the following: Consider a single left-handed standard fermion $\psi_{L 0}$ plus $p$ left-handed see-saw fermions $\Psi_{L i}$, add to it $(p+1)$ right-handed see-saw fermions $\Psi_{R j}$, and require the $(p+1) \times(p+1)$ universal see-saw mass matrix to be of the form

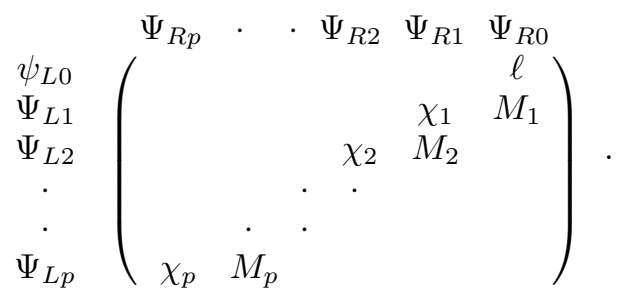

The necessary ingredients are that $\ell$ acquires the standard electroweak scale, and that the $\chi_{i}$ and $M_{j}$ are large $S U(3)_{C} \otimes S U(2)_{L} \otimes U(1)_{Y}$ invariant VEVs or bare masses with the hierarchy $\chi_{i} \ll M_{j}$. The ratios $\epsilon_{i}=\frac{\chi_{i}}{M_{i}}$ serve as hierarchy parameters in the effective low energy regime. The $p$-fold suppression of the lightest eigenvalue

$$
m_{\text {light }} \simeq \ell \epsilon_{1} \epsilon_{2} \ldots \epsilon_{p} \sim \ell \epsilon^{p}
$$

is due to the $p$-fold stairway texture of the above universal see-saw mass matrix.

The above stairway pattern is about to serve as the building block of the full Fermi mass matrix. We will consider the full mass matrix shortly, but for the moment, we need to find an underlying symmetry structure capable of supporting eq.(2.9). In the 1 -fold suppression case, discussed earlier, the underlying symmetry was Left-Right symmetry. In the $p$-fold suppression case $(p>1)$, however, a horizontal symmetry must be invoked to distinguish between the see-saw fermions in such a way that the prototype stairway pattern is produced. For a $U(1)_{H}$ symmetry to support such a pattern, the $(p+1)$ righthanded fermions involved (and separately the $p$ see-saw left-handed fermions) must have their horizontal charges form an arithmetic series. The horizontal conservation laws

$$
\begin{gathered}
L_{1}-R_{0}=\ldots=L_{p}-R_{p-1}=a \\
L_{1}-R_{1}=\ldots=L_{p}-R_{p}=b
\end{gathered}
$$

for some $a$ and $b$, result from the non-vanishing Yukawa vertices $\overline{\Psi_{L i}} M_{i} \Psi_{R(i-1)}$ and $\overline{\Psi_{L i}} \chi_{i} \Psi_{R i}$, where the horizontal charge of all the $M_{j}$ 's is $a$ and that of all the $\chi_{i}$ 's is $b$. In turn, eqs.(2.11) produce the recursion relations 


$$
\begin{aligned}
& R_{i+1}=R_{i}+(a-b) \quad \text { for all } \mathrm{i}, \\
& L_{i+1}=L_{i}+(a-b) \quad \text { for } \mathrm{i} \neq 0
\end{aligned}
$$

as a simple yet quite restrictive consequence. Such a $U(1)_{H}$ symmetry explains the stairway texture, and also provides a rationale for all the $\chi_{i}$ 's to be of the same order of magnitude (and similarly all the $M_{j}$ 's). However, it has the usual drawback of leaving the relative Yukawa coupling constants arbitrary.

This motivates for $U(1)_{H}$ to be embedded into a higher non-Abelian symmetry group. But the required embedding is obvious and natural: an $S U(2)_{H}$ symmetry can support, at no extra cost, the equally spaced horizontal charges. The $(p+1)$ right-handed see-saw fermions $\Psi_{R i}$ furnish the $(p+1)$-representation, the $p$ left-handed seesaw fermions $\Psi_{L i}$ are assigned to the $p$, whereas the single standard fermion $\psi_{L 0}$ is a singlet. The $a$ and $b$ charges are then $+1 / 2$ and $-1 / 2$, respectively, thereby suggesting that $(M, \chi)$ forms an $S U(2)_{H}$ doublet.

The real issue, however, is how to construct a mass matrix which produces one unsuppressed, one singlysuppressed, and one doubly-suppressed eigenvalue

$$
\begin{aligned}
& m_{1} \sim \ell, \\
& m_{2} \sim \ell \epsilon, \\
& m_{3} \sim \ell \epsilon^{2} .
\end{aligned}
$$

Unfortunately, this cannot be pursued by a straightforward concatenation of the $p=0,1,2$ sectors supported by $S U(2)_{H}$. Recalling that the usual universal see-saw model is Left-Right symmetric, while the generalized $p$-fold suppressing case is not, our analysis seems to (wrongly) suggest that one should abandon Left-Right symmetry, and instead use only $S U(2)_{H}$. It turns out, however, that the geometric mass hierarchy naturally hints at the larger symmetry group $S U(3)_{H}$. The point of this paper is that the universal see-saw geometric-like hierarchy, Left-Right symmetry, and the full $S U(3)_{H}$ can be reconciled with each other.

\section{Hint 3: Geometric Mass Hierarchy and $S U(3)_{H}$}

Consider a geometric-like mass hierarchy of the generic type

$$
\ell, \ell \epsilon, \ell \epsilon^{2}, \ldots, \ell \epsilon^{N-1}
$$

for an as yet unspecified number $N$ of standard families. This sequence of eigenmasses can be produced from the corresponding sequence of $p$-fold suppressing universal see-saw matrices constructed above. But the set of fermions one needs forms an interesting pattern. The right-handed see-saw fermions form the set

$$
\Psi_{R} \sim \underline{1} \oplus \underline{2} \oplus \ldots \oplus \underline{N}
$$

of $S U(2)_{H}$ representations. This immediately suggests, given the fact that the $\Psi_{R}$ 's can be conveniently grouped (for arbitrary $N$ ) within a triangular representation, the embedding of $S U(2)_{H}$ within $S U(3)_{H}$. In fact, both sets of see-saw fermions form triangles in the $\left(T_{3}, Y\right)_{H}$ plane, with the $\Psi_{L}$ triangle being one rung smaller than the $\Psi_{R}$ triangle. We therefore find a direct connection between the observed (approximate) geometric quark mass hierarchy and the appealing horizontal symmetry group $S U(3)_{H}$. It is the geometric-like Fermi mass hierarchy, and not directly the total number of families, that singles out $S U(3)_{H}$. Ironically, the standard left-handed fermions, while being $N$ in number, only form a collection of $S U(3)_{H}$ singlets.

To take stock of the situation: We have an $S U(2)_{H}$ framework that can produce any $p$-fold suppression. However, it is not left-right symmetric and there is no constraint on the number of families. It can incorporate the observed geometric-like pattern of fermion masses, but it does not predict this pattern uniquely. However, if the eigenmass sequence is taken as geometric, then the pattern of $S U(2)_{H}$ fermion representations strongly suggests an underlying $S U(3)_{H}$. The number of standard left-handed fermions is, however, still not constrained by the 'horizontal' symmetry. The pieces of the puzzle almost fit.

The theory we describe in the next section dovetails the geometric universal see-saw mass hierarchy, horizontal $S U(3)_{H}$ and Left-Right symmetry. The resulting fermion mass matrix has an intricate texture in which a Left-Right symmetric geometric sequence of stairway patterns can be discerned, but not in the obvious, naive way. The key to resolving the apparent conflict between Left-Right symmetry and the geometric sequence of $p$ fold universal see-saw suppression is the following set of five building blocks

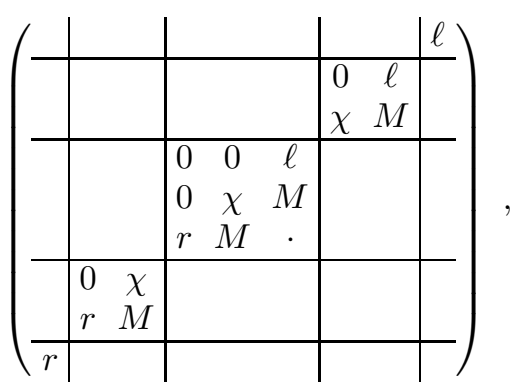

generalizing the three building block toy set eq.(2.8). Note that the "." entry in the middle block can be as large as $M$ without spoiling the lightest eigenvalue pattern. This sequence is manifestly Left-Right symmetric and geometric-like. It extends the pattern of the toy model eq.(2.8), but inserts a novel feature $\chi$ whose significance will be explained below. The full mass matrix thus has dimensionality $1+2+3+2+1=9$.

Which features of the three hints are to be retained and which abandoned? The answer to this question is not $a$ priori obvious. We now present a scheme that works. 


\section{LEFT-RIGHT SYMMETRIC $S U(3)_{H}$ THEORY OF THE GEOMETRIC MASS HIERARCHY}

\section{Symmetry Group and Multiplet Assignments:}

The largest representation suggested by hint 3, namely $\frac{1}{2} N(N+1)$, is maintained for the right-handed see-saw fermions $\Psi_{R}$. In keeping with our demand of Left-Right symmetry, the left-handed see-saw fermions $\Psi_{L}$ can now be either the vector-like choice $\frac{1}{2} N(N+1)$ or the flavorchiral choice $\underline{\frac{1}{2} N(N+1)^{*}}$. This is of course a departure from hint 3 , which would require a $\frac{1}{2} N(N-1)$ or $\frac{1}{2} N(N-1)^{*}$. For reasons to be specified soon, we choose the flavor-chiral option rather than the vector-like option. Next, consider the $N$ left-handed and the $N$ right-handed standard fermions, $\psi_{L}$ and $\psi_{R}$ respectively. Each must constitute some, preferably irreducible, representation. We adopt the simplest non-trivial choice of either the fundamental representation or its conjugate (again departing from hint 3 ). Since we already know from the geometric-like mass hierarchy that the horizontal group is $S U(3)_{H}$, this forces $N=3$. In this way, the total number of standard families gets correlated with the geometric-like Fermi mass hierarchy. We resolve the conjugation ambiguity by choosing the "same triality" assignments

$$
\begin{aligned}
& \psi_{L} \oplus \Psi_{L} \sim \underline{3} \oplus \underline{6}^{*}, \\
& \psi_{R} \oplus \Psi_{R} \sim \underline{3}^{*} \oplus \underline{6} .
\end{aligned}
$$

The combined requirements of flavor-chirality and same triality ensure that a group theoretically attractive Higgs sector can couple to fermions through Yukawa interactions (see later). The flavor-chiral option is also favored on electro/nuclear unification grounds.

We now specify the multiplet structure of our theory. Using $\left(S U(3)_{H} \mid S U(3)_{C} \otimes S U(2)_{L} \otimes S U(2)_{R}\right)_{B-L}$ notation, our scheme is spanned by the Fermi sector

$$
\begin{aligned}
& q_{L}(\underline{3} \mid \underline{3}, \underline{2}, \underline{1})_{+\frac{1}{3}}+Q_{L}^{u, d}\left(\underline{6}^{*} \mid \underline{3}, \underline{1}, \underline{1}\right)_{+\frac{4}{3},-\frac{2}{3}} \\
& q_{R}\left(\underline{3}^{*} \mid \underline{3}, \underline{1}, \underline{2}\right)_{+\frac{1}{3}}+Q_{R}^{u, d}(\underline{6} \mid \underline{3}, \underline{1}, \underline{1})_{+\frac{4}{3},-\frac{2}{3}}
\end{aligned}
$$

accompanied by leptons, and the Higgs sector

$$
\begin{gathered}
\ell_{u, d}(\underline{3} \mid \underline{1}, \underline{2}, \underline{1})_{+1,-1}+r_{u, d}(\underline{3} \mid \underline{1}, \underline{1}, \underline{2})_{-1,+1} \\
\Phi_{u, d}\left(\underline{6}^{*} \mid \underline{1}, \underline{1}, \underline{1}\right)_{0,0} .
\end{gathered}
$$

Several remarks are in order:

1. All left-handed fermions (and anti-fermions) and Higgs scalars transform horizontally alike, namely via $\underline{3} \oplus \underline{6}^{*}$. The Yukawa interactions are given by

$$
\begin{aligned}
& \mathcal{L}_{Y}=\left[\lambda_{u}\left(\bar{q}_{L} Q_{R}^{u} \ell_{u}^{*}+\bar{q}_{R} Q_{L}^{u} r_{u}\right)+\Lambda_{u} \bar{Q}_{R}^{u} Q_{L}^{u} \Phi_{u}\right]+ \\
& +\left[\lambda_{d}\left(\bar{q}_{L} Q_{R}^{d} \ell_{d}^{*}+\bar{q}_{R} Q_{L}^{d} r_{d}\right)+\Lambda_{d} \bar{Q}_{R}^{d} Q_{L}^{d} \Phi_{d}\right]+\text { h.c },
\end{aligned}
$$

where the charge $+2 / 3$ sector terms are grouped between the first pair of square brackets, and the charge $-1 / 3$ sector between the second pair. The $S U(3)_{H}$ fermion assignments selected allow, through $S U(3)$ group theory, the parallel structure for the Higgs bosons.

2. The marriage of the universal see-saw mechanism, Left-Right symmetry, and horizontal $S U(3)_{H}$ is schematically expressed, in the Yukawa sector, by correlating

$$
(\underline{2}, \underline{1}) \quad\left(\begin{array}{c|c}
(\underline{1}, \underline{2}) & (\underline{1}, \underline{1}) \\
(\underline{1}, \underline{1}) & (\underline{2}, \underline{1}) \\
\hline(\underline{1}, \underline{2}) & (\underline{1}, \underline{1})
\end{array}\right)
$$

with

$$
\underline{6}^{*} \quad\left(\begin{array}{c|c}
\underline{3} & \underline{6}^{*} \\
\hline \underline{3} & \underline{3}^{*} \\
\hline \underline{6} & \underline{6}^{*}
\end{array}\right),
$$

owing its simplicity to the fact that $\underline{3} \times \underline{6}^{*} \times \underline{6}^{*} \not \supset \underline{1}$. While being a necessary condition for the universal seesaw mechanism, the absence of the $\left(\underline{3} \oplus \underline{6}^{*} \mid \underline{2}, \underline{2}\right)$ entry from the standard electroweak corner is not a sufficient condition for establishing the correct mass hierarchy. The latter calls for a certain detailed structure at the $\left(\underline{6}^{*} \mid \underline{1}, \underline{1}\right)$ corner. It must be singular enough to support exactly one unsuppressed eigenmass, and patterned in a particular manner in order to produce a full geometric-like hierarchy.

3. The possibility of a see-saw sector analogue of $S U(2)_{L} \otimes S U(2)_{R}$ is strongly suggested by the assignments in eq.(3.2). All fermions and scalars in our scheme fall naturally into $S U(2)_{L}^{\prime} \otimes S U(2)_{R}^{\prime}$ representations: $Q_{L}^{u, d}$ and $r_{u, d}$ can form $\left(\underline{2}^{\prime}, \underline{1}^{\prime}\right)$ multiplets, $Q_{R}^{u, d}$ and $\ell_{u, d}$ can form $\left(\underline{1}^{\prime}, \underline{2}^{\prime}\right)$ multiplets, while $\Phi_{u, d}$ can live in $\left(\underline{2}^{\prime}, \underline{2}^{\prime}\right)$. In this way, the see-saw sector weakisospin gauge group can elegantly explain why the electric charges of see-saw matter match those of standard matter. Furthermore, it motivates an electro/nuclear $\left[S O(10) \otimes S O(10)^{\prime}\right] \otimes S U(3)_{H}$ unification scheme.

4. The doubling of scalars into an up-type and a downtype, dictated by the horizontal flavor chirality for the Higgs triplets, is recognized as a vital ingredient in any supersymmetric [9] extension of the model. Note that the doubling of Higgs sextets $\Phi$ is dictated by gauge symmetry only when the $S U(2)_{L}^{\prime} \otimes S U(2)_{R}^{\prime}$ extension is invoked.

5. The flavor-chiral nature of $S U(3)_{H}$ suggests that the full theory contains an $S U(3)_{H}$ anomaly, in which case the horizontal symmetry must be global rather than local. The spontaneous breakdown of global $S U(3)_{H}$ has interesting consequences in regard to familon [10] phenomenology and large-scale structure formation in cosmology 11.

\section{Mass Matrix and VEV Pattern:}

Having discussed the important features of the multiplet assignments, we note that, while elegant, they do 
not of themselves lead to the desired geometric-like hierarchical structure. A particular VEV pattern is also necessary. To fully appreciate this, we must first examine the general $9 \times 9$ mass matrix that arises from the Yukawa Lagrangian eq.(3.4). Since the mass matrix is of the same form in both of the quark sectors, we can momentarily consider the generic mass matrix $\mathcal{M}$ :

$$
\left(\begin{array}{ccc|cccccc}
0 & 0 & 0 & \ell^{1} & 0 & 0 & 0 & \frac{\ell^{3}}{\sqrt{2}} & \frac{\ell^{2}}{\sqrt{2}} \\
0 & 0 & 0 & 0 & \ell^{2} & 0 & \frac{\ell^{3}}{\sqrt{2}} & 0 & \frac{\ell^{1}}{\sqrt{2}} \\
0 & 0 & 0 & 0 & 0 & \ell^{3} & \frac{\ell^{2}}{\sqrt{2}} & \frac{\ell^{1}}{\sqrt{2}} & 0 \\
\hline r^{1} & 0 & 0 & 0 & \Phi_{33} & \Phi_{22} & \Phi_{23} & 0 & 0 \\
0 & r^{2} & 0 & \Phi_{33} & 0 & \Phi_{11} & 0 & \Phi_{13} & 0 \\
0 & 0 & r^{3} & \Phi_{22} & \Phi_{11} & 0 & 0 & 0 & \Phi_{12} \\
0 & \frac{r^{3}}{\sqrt{2}} & \frac{r^{2}}{\sqrt{2}} & \Phi_{23} & 0 & 0 & \Phi_{11} & -\frac{\Phi_{12}}{\sqrt{2}} & -\frac{\Phi_{13}}{\sqrt{2}} \\
\frac{r^{3}}{\sqrt{2}} & 0 & \frac{r^{1}}{\sqrt{2}} & 0 & \Phi_{13} & 0 & -\frac{\Phi_{12}}{\sqrt{2}} & \Phi_{22} & -\frac{\Phi_{23}}{\sqrt{2}} \\
\frac{r^{2}}{\sqrt{2}} & \frac{r^{1}}{\sqrt{2}} & 0 & 0 & 0 & \Phi_{12} & -\frac{\Phi_{13}}{\sqrt{2}} & -\frac{\Phi_{23}}{\sqrt{2}} & \Phi_{33}
\end{array}\right) .
$$

The various entries denote vacuum expectation values multiplied by Yukawa coupling constants. These entries are labeled by the corresponding Higgs multiplet components. Thus,

$$
\begin{aligned}
\left(\ell^{1}, \ell^{2}, \ell^{3}\right) & =\lambda\langle\ell\rangle, \\
\left(r^{1}, r^{2}, r^{3}\right) & =\lambda\langle r\rangle, \\
\left(\Phi_{i j}\right) & =\Lambda\langle\Phi\rangle .
\end{aligned}
$$

The first three rows are multiplied by the three horizontal components of $\bar{q}_{L}(q=u, d)$ in the order 1, 2, 3; the second three rows by the diagonal (11), (22) and (33) components of $\bar{Q}_{L}^{q}$; and the last three rows by the offdiagonal (23), (13) and (12) components of $\bar{Q}_{L}^{q}$, where the sextet is denoted by a $3 \times 3$ symmetric matrix. The columns are multiplied by the components of $q_{R}$ and $Q_{R}^{q}$ in the corresponding order. Notice the Clebsch-Gordan coefficients $\pm 1, \pm \frac{1}{\sqrt{2}}$; they play an important role in the model.

We take the left-handed and right-handed horizontal triplet VEVs to be aligned. This means that we can use a horizontal rotation to transform any two components to zero. We choose

$$
\begin{aligned}
& \ell^{1,3}=0, \\
& r^{1,3}=0,
\end{aligned}
$$

and denote

$$
\begin{aligned}
\ell^{2} & \equiv \ell, \\
r^{2} & \equiv r .
\end{aligned}
$$

Notice that the triplet VEVs exhibit a residual $S U(2)_{H}$ symmetry in the (1,3)-plane. An $S U(2)_{H}$ transformation $\Phi \rightarrow U \Phi U^{T}$ allows us then to set $\Phi_{13}=\Phi_{31}=0$, so that

$$
\Phi_{i j} \equiv\left(\begin{array}{ccc}
z & v & 0 \\
v & x & y \\
0 & y & t
\end{array}\right)
$$

The generic mass matrix $\mathcal{M}$ becomes

$$
\mathcal{M}=\left(\begin{array}{ccccc|cccc}
0 & 0 & 0 & 0 & 0 & 0 & 0 & 0 & \frac{1}{\sqrt{2}} \ell \\
0 & 0 & 0 & 0 & \ell & 0 & 0 & 0 & 0 \\
0 & 0 & 0 & 0 & 0 & 0 & \frac{1}{\sqrt{2}} \ell & 0 & 0 \\
0 & 0 & 0 & 0 & -t & -x & -y & 0 & 0 \\
0 & r & 0 & -t & 0 & -z & 0 & 0 & 0 \\
\hline 0 & 0 & 0 & -x & -z & 0 & 0 & 0 & -v \\
0 & 0 & \frac{1}{\sqrt{2}} r & -y & 0 & 0 & z & \frac{1}{\sqrt{2}} v & 0 \\
0 & 0 & 0 & 0 & 0 & 0 & \frac{1}{\sqrt{2}} v & x & \frac{1}{\sqrt{2}} y \\
\frac{1}{\sqrt{2}} r & 0 & 0 & 0 & 0 & -v & 0 & \frac{1}{\sqrt{2}} y & t
\end{array}\right) .
$$

The sextet VEV pattern is dominated by a heavy mass scale $M$. But which entries of $\Phi_{i j}$ actually carry this scale? Clearly, they cannot all share such a property, as otherwise all light eigenmasses get suppressed. Indeed, the requirement of having one and only one family with eigenmass $\sim \ell$, a vital mass hierarchy ingredient, severely constrains the tenable VEV pattern. To see the point, notice that for any given $\ell$ in the generic mass matrix (3.13) to be suppressed at low energies, at least one entry along its column must be $\sim M$. Up to a residual $S U(2)_{H}$ rotation, this leaves us with only two options to consider: (i) Either $v \sim M$ and $t, y, z \ll M$, leading to

$$
\Phi_{i j}=\left(\begin{array}{ccc}
0 & M & 0 \\
M & x & 0 \\
0 & 0 & 0
\end{array}\right)+\ldots
$$

(ii) or $z \sim M$ and $v, y, t \ll M$, for which

$$
\Phi_{i j}=\left(\begin{array}{ccc}
M & 0 & 0 \\
0 & x & 0 \\
0 & 0 & 0
\end{array}\right)+\ldots .
$$

Although the value of $x$ is not directly restricted, it cannot nonetheless to be $\sim M$, as otherwise another $\mathcal{O}(\ell)$ eigenmass $\sim \frac{x \ell}{\sqrt{M^{2}+x^{2}}}$ would make its appearance; this refers to both options. Also, the second option can be rejected on phenomenological grounds. It lacks the stairway structure eq.(2.9), and thus is not capable of producing a doubly suppressed light eigenmass. One thus finally deduces that the sextet VEV pattern must be governed by

$$
\Phi_{i j}=\left(\begin{array}{ccc}
0 & M & 0 \\
M & 0 & 0 \\
0 & 0 & 0
\end{array}\right)+\ldots
$$

We now look again at eq.(3.13) where the dominant entry $v$ will now be relabeled as $M$, and focus on the effects 
of the sub-dominant $x, y, z, t$-entries. The geometrically hierarchical stairway texture is evident. To be more specific, here are the see-saw sub-matrices that are primarily responsible for the geometric mass hierarchy:

1. The $\ell$ entry in row-2 (and similarly the $r$ entry in column-2),

$$
\mathcal{M}_{25}=\ell\left(\mathcal{M}_{52}=r\right),
$$

does not meet any heavy $M$-entry along its column (row) and thus stay unsuppressed.

2 . The $\frac{1}{\sqrt{2}} \ell$ entry in row- 1 , and similarly the $\frac{1}{\sqrt{2}} r$ entry in column-1, are involved in the $2 \times 2$ see-saw sub-matrices

$$
\begin{aligned}
& \left(\begin{array}{ll}
\mathcal{M}_{14} & \mathcal{M}_{19} \\
\mathcal{M}_{64} & \mathcal{M}_{69}
\end{array}\right)=\left(\begin{array}{cc}
0 & \frac{1}{\sqrt{2}} \ell \\
-x & -M
\end{array}\right), \\
& \left(\begin{array}{ll}
\mathcal{M}_{41} & \mathcal{M}_{46} \\
\mathcal{M}_{91} & \mathcal{M}_{96}
\end{array}\right)=\left(\begin{array}{cc}
0 & -x \\
\frac{1}{\sqrt{2}} r & -M
\end{array}\right) .
\end{aligned}
$$

These give rise to singly suppressed eigenmasses $\frac{x \ell}{\sqrt{2} M}$ and $\frac{x r}{\sqrt{2} M}$, respectively.

3 . The $\frac{1}{\sqrt{2}} \ell$ entry of row- 3 together with the $\frac{1}{\sqrt{2}} r$ entry of row -7 participate in the $3 \times 3$ Left-Right symmetric see-saw sub-matrix

$$
\left(\begin{array}{lll}
\mathcal{M}_{33} & \mathcal{M}_{38} & \mathcal{M}_{37} \\
\mathcal{M}_{83} & \mathcal{M}_{88} & \mathcal{M}_{87} \\
\mathcal{M}_{73} & \mathcal{M}_{78} & \mathcal{M}_{77}
\end{array}\right)=\left(\begin{array}{ccc}
0 & 0 & \frac{1}{\sqrt{2}} \ell \\
0 & x & \frac{1}{\sqrt{2}} M \\
\frac{1}{\sqrt{2}} r & \frac{1}{\sqrt{2}} M & z
\end{array}\right) .
$$

The corresponding light eigenmass is $\frac{x r \ell}{M^{2}}$.

So far, we have determined that the mass hierarchy parameters are $\frac{r}{M}$ and $\frac{x}{M}$. But, we have to yet gain insight into the significance of $y, z, t$. To do so, we need to discuss the mixing angle hierarchies of the CabibboKobayashi-Maskawa (CKM) matrix. To proceed, we set

$$
M \gg x, y, z \gg t,
$$

and evaluate the effective $5 \times 5$ mass matrix $\mathcal{M}_{L R}$ obtained by "integrating out" the four $\mathcal{O}(M)$-states. On phenomenological grounds, we must require $t$ to be very small (see below). Note that the parameter $t$ is special because it appears in the effective $5 \times 5$ matrix directly. This is easy to verify from eq.(3.13), by noticing that the bottom-right $4 \times 4$ corner contains the four heavy states. The $M \rightarrow \infty$ limit leads to the removal of all the rows and columns containing the $M$ entry; in this process, the parameters $x, y, z$ also get removed, but $t$ remains.

The $5 \times 5$ effective mass matrix is given by

$$
\mathcal{M}_{L R}=\left(\begin{array}{cc|ccc}
0 & 0 & 0 & 0 & \ell \\
0 & 0 & 0 & -\frac{1}{\sqrt{2}} \ell \frac{x}{M} & -\frac{1}{\sqrt{2}} \ell \frac{z}{M} \\
0 & 0 & -\ell \frac{r x}{M^{2}} & \frac{1}{\sqrt{2}} \ell \frac{x y}{M^{2}} & -\frac{1}{\sqrt{2}} \ell \frac{y z}{M^{2}} \\
\hline 0-\frac{1}{\sqrt{2}} r \frac{x}{M} & \frac{1}{\sqrt{2}} r \frac{x y}{M^{2}} & \ldots & t+\frac{y^{2} z}{M^{2}} \\
r & -\frac{1}{\sqrt{2}} r \frac{z}{M} & -\frac{1}{\sqrt{2}} r \frac{y z}{M^{2}} & t+\frac{y^{2} z}{M^{2}} & \ldots
\end{array}\right)
$$

where the "..." entries are zero to $\mathcal{O}\left(1 / M^{2}\right)$. The heavy fermions $Q^{33}, Q^{12}, Q^{23}, Q^{31}$ have been decoupled. Thus, up to $\mathcal{O}(1 / M)$ corrections, the fermionic basis of $\mathcal{M}_{L R}$ consists of $\left(q_{2}, q_{1}, q_{3}, Q^{11}, Q^{22}\right)$ [note that $q_{1}$ and $q_{2}$ have for convenience been interchanged with respect to eq. 3.13)].

A number of observations need to be made:

1. The effective mass matrix $\mathcal{M}_{L R}$ displays the remnants of Left-Right symmetry (broken only because $\ell \neq r$ ).

2. The eigenvalues are given by the magnitudes of the anti-diagonal entries [provided that (3) below holds],

$$
\ell, \frac{\ell}{\sqrt{2}} \frac{x}{M}, \ell r \frac{x}{M^{2}}, \frac{r}{\sqrt{2}} \frac{x}{M}, r .
$$

3. We need the potentially large term

$$
t+\frac{y^{2} z}{M^{2}} \simeq 0 .
$$

If this term is too large, the would-be $\mathcal{O}(\ell)$ eigenvalue gets see-saw suppressed.

4. With eq.(3.23) in place, the effective $3 \times 3$ mass matrix $\mathcal{M}_{\text {eff }}$ pertaining to the $r \gg \ell$ limit is the upper-right $3 \times 3$ block

$$
\mathcal{M}_{\text {eff }}=\left(\begin{array}{ccc}
0 & 0 & \ell \\
0 & -\frac{1}{\sqrt{2}} \ell \frac{x}{M} & -\frac{1}{\sqrt{2}} \ell \frac{z}{M} \\
-\ell \frac{r x}{M^{2}} & \frac{1}{\sqrt{2}} \ell \frac{x y}{M^{2}} & -\frac{1}{\sqrt{2}} \ell \frac{y z}{M^{2}}
\end{array}\right) .
$$

5. Note the unusual fermion basis of $\mathcal{M}_{\text {eff }}$. The righthanded partners of the mostly 'standard' left-handed quarks $\left(q_{2 L}, q_{1 L}, q_{3 L}\right)$ are $\left(Q_{R}^{22}, Q_{R}^{11}, q_{3 R}\right)$, respectively, two of which are mostly 'see-saw' fermions. The lightest family is therefore predicted to have different $S U(2)_{R}$ gauge couplings compared to the heavier families.

6. The light family is also special in another respect in our scheme, because it is its own $\ell \leftrightarrow r$ partner.

7. The left-sector mixing angles are governed by the ratios $\frac{z}{M}$ and $\frac{y}{M}$. To improve predictivity, we would like the mixing parameters to be related to the mass hierarchy parameters. To this end we introduce the requirement that

$$
|x| \simeq|z|
$$

So, we have identified the requirements eqs. (3.23, 3.25) as phenomenological necessities within the general sextet VEV pattern. Is there something mathematically significant about these phenomenological constraints? Quite remarkably, there is a simple geometric underpinning. The required VEV pattern for each quark sector can be produced by

$$
\begin{aligned}
& <\underline{3}>=(\ell \text { or } r)\left(\begin{array}{l}
0 \\
1 \\
0
\end{array}\right), \\
& <\underline{6}^{*}>=U\left(\begin{array}{ccc}
0 & M & 0 \\
M & 0 & 0 \\
0 & 0 & 0
\end{array}\right) U^{T},
\end{aligned}
$$


where $U$ is some $S U(3)_{H}$ matrix. In other words, the realistic sextet patterns are just $S U(3)_{H}$ transformations of the dominant pattern. The charge $2 / 3$ and $-1 / 3$ sectors differ from each other by their relative scales $\ell_{u, d} \ll r_{u, d} \ll M$ and by the $S U(3)_{H}$ rotations $U_{u, d}$. The departure of the matrices $U_{u, d}$ from the identity is crucial for generating the mass hierarchy (through the $x$-parameter), and for producing the CKM mixings (through $y$ and $z$ ).

The requirement $\Phi_{13}=\Phi_{31}=0$, see eq.(3.12), is translated into

$$
U_{11} U_{32}+U_{12} U_{31}=0 .
$$

The most general $S U(3)_{H}$ matrix (up to diagonal phase matrices from its left and/or right) that satisfies this takes the form

$$
U=\left(\begin{array}{ccc}
\cos \frac{\tilde{h}}{2} \cos \alpha & -\sin \frac{h}{2} \cos \alpha & . \\
\sin \frac{\tilde{h}}{2} & \cos \frac{h}{2} & . \\
\cos \frac{\tilde{h}}{2} \sin \alpha & \sin \frac{h}{2} \sin \alpha & .
\end{array}\right),
$$

subject to the unitarity constraint

$$
\cos 2 \alpha=\frac{\tan \frac{\tilde{h}}{2}}{\tan \frac{h}{2}} .
$$

The "." entries, calculable by means of unitarity, are of no relevance to our discussion. The quantities $h$ and $\tilde{h}$ are mass hierarchy parameters, while the mixing parameter $\alpha$ is related to the Cabibbo angle. Remembering that the Cabibbo angle is not too small, we note that the unitarity constraint allows $\alpha$ to stay finite in the limit $\tilde{h} \leq h \rightarrow 0$. To the leading order in $h$ and $\tilde{h}$, and for arbitrary $\alpha$,

$$
<\Phi>\simeq M\left(\begin{array}{ccc}
-h \cos ^{2} \alpha & \cos \alpha & 0 \\
\cos \alpha & h \cos 2 \alpha & \sin \alpha \\
0 & \sin \alpha & h \sin ^{2} \alpha
\end{array}\right) .
$$

This pattern is characterized by

$$
t+\frac{y^{2} z}{M^{2} \cos ^{2} \alpha}=\mathcal{O}\left(h^{3}\right),
$$

which is very small (note that $M \cos \alpha$ now plays the role of $M)$. Furthermore, the pattern is traceless,

$$
z+x+t=0,
$$

and if $\alpha$ itself is first-order, we observe that

$$
z+x \simeq \mathcal{O}\left(h \alpha^{2}\right),
$$

which is also small. The phenomenological constraints eq.(3.23) and eq.(3.25) are thus satisfied in a non-trivial way.

Substituting the VEV pattern eq.(3.30) into the lowenergy effective mass matrix $\mathcal{M}_{\text {eff }}$, we calculate the corresponding CKM matrix $V_{C K M}$ to find that

$$
\begin{aligned}
& V_{11} \simeq \cos \left(\alpha_{d}-\alpha_{u}\right), \\
& V_{22} \simeq \cos \left(\alpha_{d}-\alpha_{u}\right), \\
& V_{33} \simeq 1 \\
& V_{12} \simeq \sin \left(\alpha_{d}-\alpha_{u}\right) \\
& V_{21} \simeq-\sin \left(\alpha_{d}-\alpha_{u}\right) \\
& V_{23} \simeq \frac{1}{\sqrt{2}}\left[-h_{u} \cos 2 \alpha_{u}+h_{d} \cos \left(\alpha_{d}+\alpha_{u}\right)\right], \\
& V_{32} \simeq \frac{1}{\sqrt{2}}\left[-h_{d} \cos 2 \alpha_{d}+h_{u} \cos \left(\alpha_{d}+\alpha_{u}\right)\right], \\
& V_{13} \simeq \frac{1}{\sqrt{2}}\left[h_{u} \sin 2 \alpha_{u}-h_{d} \sin \left(\alpha_{d}+\alpha_{u}\right)\right], \\
& V_{31} \simeq \frac{1}{\sqrt{2}}\left[h_{d} \sin 2 \alpha_{d}-h_{u} \sin \left(\alpha_{d}+\alpha_{u}\right)\right] .
\end{aligned}
$$

The qualitative structure of $V_{C K M}$ is correct: $V_{12}$ is suppressed with respect to the diagonal, $V_{23}$ and $V_{13}$ are suppressed by the mass hierarchy parameters $h_{u, d}$, and $V_{13}$ is smaller than $V_{23}$ by a factor of the order of the Cabibbo angle.

Before proceeding to discuss mass and mixing angle relationships in detail, let us re-emphasize the geometric origin of the hierarchy parameters $h_{u, d}$ and the mixing parameters $\alpha_{u, d}$. They arise by applying $S U(3)_{H}$ transformations on a simple primordial $\langle\Phi\rangle$ form. The hierarchies are then due to these transformations $U_{u, d}$ being only slightly different from the identity. This suggests that the observed pattern of quark mass and mixing, despite its complexity, may reflect a simple geometric structure in an internal horizontal space.

\section{Mass and Mixing Angle Relations:}

The preceding subsection revealed the VEV pattern required for our scheme to work. It also provided a surprising geometric interpretation for this pattern. Two important questions, which appear to be intertwined, are now in order: Is our theory quantitatively as well as qualitatively successful? What is the origin of the VEV pattern?

From eq.(3.34), we see that

$$
V_{12} \simeq-V_{21} \simeq \alpha_{d}-\alpha_{u}
$$

Interestingly, within the framework of our geometric mass hierarchy model, the Cabibbo angle

$$
\theta_{c}=\alpha_{d}-\alpha_{u} \simeq 0.22,
$$

has nothing to do with the mass hierarchy. The hierarchy and the mixing parameters may get correlated, however, by means of the Higgs potential.

Assuming that $\alpha_{u, d} \ll 1$, the hierarchy parameters can be easily identified

$$
\begin{aligned}
& h_{u} \simeq \pm \sqrt{2} \frac{m_{c}}{m_{t}}, \\
& h_{d} \simeq \pm \sqrt{2} \frac{m_{s}}{m_{b}},
\end{aligned}
$$

where the sign ambiguities remain unresolved at this level. Equation (3.34) then produces the first indication of a mass and mixing angle relationship, 


$$
V_{23} \simeq-V_{32} \simeq \frac{1}{\sqrt{2}}\left(h_{d}-h_{u}\right) \simeq \frac{m_{s}}{m_{b}} \pm \frac{m_{c}}{m_{t}} .
$$

On phenomenological grounds, we need the relative sign between the mass ratios to be positive (see below).

The light-heavy mixing entries are given by

$$
\begin{aligned}
& V_{13} \simeq-\frac{1}{\sqrt{2}} h_{d}\left(\alpha_{u}+\alpha_{d}\right)+\sqrt{2} h_{u} \alpha_{u}, \\
& V_{31} \simeq-\frac{1}{\sqrt{2}} h_{u}\left(\alpha_{u}+\alpha_{d}\right)+\sqrt{2} h_{d} \alpha_{d} .
\end{aligned}
$$

A second phenomenologically successful relationship is then

$$
V_{13}+V_{31} \simeq \frac{1}{\sqrt{2}}\left(h_{d}-h_{u}\right)\left(\alpha_{d}-\alpha_{u}\right) \simeq V_{12} V_{23},
$$

involving mixing angles only (at this level of approximation), and independent of the sign ambiguities.

To proceed, we need more information about the hierarchy parameters $h_{u, d}$ and the mixing parameters $\alpha_{u, d}$. The obvious place to seek this information is from the minimization of the Higgs potential. Unfortunately, the full Higgs potential is very complicated, making it difficult to extract insight. We can obtain very interesting results, however, from a partial analysis, which we now present.

The full Higgs potential contains all renormalizable, gauge and $S U(3)_{H}$ invariant terms involving the Higgs multiplets $\ell_{u, d}, r_{u, d}$ and $\Phi_{u, d}$. These terms fall into two classes: those that are sensitive to the internal structure of the Higgs multiplets, and those that only depend on the overall scales of the Higgs multiplets. Our partial analysis has the following restrictions:

(i) It focuses on the internal structure sensitive class.

(ii) It assumes that the horizontal triplet VEVs are fixed in the $(0,1,0)$ configuration.

(iii) It assumes that the sextet VEV pattern is of the form required by the geometric ansatz.

(iv) It invokes, for simplicity, constraints motivated by a possible $S U(2)_{L}^{\prime} \otimes S U(2)_{R}^{\prime}$ see-saw weak isospin gauge group.

The Higgs potential, with the above restrictions incorporated, can be written in the form

$$
V=V_{0}+V_{\text {int }}\left(\alpha_{u}, \alpha_{d}, h_{u}, h_{d}\right)
$$

where

$$
\begin{aligned}
& V_{\text {int }}=-\frac{1}{4} a_{1} \operatorname{Tr}\left(\Phi_{u}^{\dagger} \Phi_{d}\right) \operatorname{Tr}\left(\Phi_{d}^{\dagger} \Phi_{u}\right)- \\
& -a_{2} \operatorname{Tr}\left(\Phi_{u}^{\dagger} \Phi_{d} \Phi_{d}^{\dagger} \Phi_{u}\right)+ \\
& +b\left\langle r_{u}^{i}\right\rangle\left\langle r_{d}^{i^{\prime}}\right\rangle \epsilon^{i j k} \epsilon^{i^{\prime} j^{\prime} k^{\prime}} \Phi_{j k^{\prime}} \Phi_{j^{\prime} k}+ \\
& +c_{1}\left[\left\langle r_{d}\right\rangle^{\dagger} \Phi_{u}^{\dagger} \Phi_{u}\left\langle r_{d}\right\rangle+\left\langle r_{u}\right\rangle^{\dagger} \Phi_{d}^{\dagger} \Phi_{d}\left\langle r_{u}\right\rangle\right]+ \\
& +c_{2}\left[\left\langle r_{u}\right\rangle^{\dagger} \Phi_{u}^{\dagger} \Phi_{u}\left\langle r_{u}\right\rangle+\left\langle r_{d}\right\rangle^{\dagger} \Phi_{d}^{\dagger} \Phi_{d}\left\langle r_{d}\right\rangle\right]+ \\
& +(r \rightarrow \ell) .
\end{aligned}
$$

Expanding up to fourth order in $\alpha$ and $h$, we obtain

$$
\begin{aligned}
& V_{i n t} \simeq\left(a_{1}+a_{2}\right)\left[\left(\alpha_{u}-\alpha_{d}\right)^{2}+\frac{1}{3}\left(\alpha_{u}-\alpha_{d}\right)^{4}\right]+ \\
& +2\left[a_{2}\left(1-3 \alpha_{u}^{2}-\alpha_{d}^{2}+\alpha_{u} \alpha_{d}\right)+a_{1} \alpha_{u} \alpha_{d}+2 p_{u} \alpha_{u}^{2}\right] h_{u}^{2}+ \\
& +2\left[a_{2}\left(1-\alpha_{u}^{2}-3 \alpha_{d}^{2}+\alpha_{u} \alpha_{d}\right)+a_{1} \alpha_{u} \alpha_{d}+2 p_{d} \alpha_{d}^{2}\right] h_{d}^{2}- \\
& -4\left[a_{2}\left(1-2 \alpha_{u}^{2}-2 \alpha_{d}^{2}+\alpha_{u} \alpha_{d}\right)+\right. \\
& \left.+a_{1} \alpha_{u} \alpha_{d}+q\left(\alpha_{u}^{2}+\alpha_{d}^{2}\right)\right] h_{u} h_{d}-\frac{4}{3} a_{2}\left(h_{d}-h_{u}\right)^{4}
\end{aligned}
$$

where

$$
\begin{aligned}
q & \equiv \frac{1}{2} b r_{u} r_{d}, \\
p_{u} & \equiv-\frac{1}{2}\left(c_{1} r_{d}^{2}+c_{2} r_{u}^{2}\right), \\
p_{d} & \equiv-\frac{1}{2}\left(c_{1} r_{u}^{2}+c_{2} r_{d}^{2}\right),
\end{aligned}
$$

having inputted $|r| \gg|\ell|$.

To minimize this potential, we keep the $\alpha$ 's and $h$ 's small, and identify a region of parameter space that is phenomenologically interesting. It turns out that the relevant region in parameter space is where the $p$ 's dominate the quartic couplings

$$
\left|p_{u, d}\right| \gg|q|,\left|a_{1,2}\right|,
$$

leading to

$$
\begin{aligned}
V_{i n t} & \simeq\left(a_{1}+a_{2}\right)\left(\alpha_{u}-\alpha_{d}\right)^{2}+2 a_{1}\left(h_{u}-h_{d}\right)^{2}+ \\
& +4\left(p_{u} \alpha_{u}^{2} h_{u}^{2}+p_{d} \alpha_{d}^{2} h_{d}^{2}\right) .
\end{aligned}
$$

Two of the four minimization equations then lead to the remarkable relations

$$
\frac{h_{u}}{h_{d}}=\frac{\alpha_{u}}{\alpha_{d}}=-\left(\frac{p_{d}}{p_{u}}\right)^{1 / 3} .
$$

Given that the ratio of the $p$ 's is positive (as the potential is bounded from below), one very important consequence is that

$$
\frac{h_{u}}{h_{d}}<0 .
$$

This resolves the sign ambiguity of eq.(3.38), giving rise to the phenomenologically successful relation

$$
V_{23} \simeq-V_{32} \simeq \frac{m_{s}}{m_{b}}+\frac{m_{c}}{m_{t}} .
$$

Combining eq.(3.47) with the $\theta_{c}$ expression, we get

$$
\begin{aligned}
\alpha_{d} & =\frac{h_{d}}{h_{d}-h_{u}} \theta_{c}, \\
\alpha_{u} & =\frac{h_{u}}{h_{d}-h_{u}} \theta_{c} .
\end{aligned}
$$

Substituting these results into the light-heavy mixing terms, we can now obtain individual relations for $V_{13}$ and $V_{31}$, namely 


$$
\begin{aligned}
& V_{13} \simeq \frac{1}{\sqrt{2}}\left(h_{d}+2 h_{u}\right) \theta_{c} \simeq\left(2 \frac{m_{c}}{m_{t}}-\frac{m_{s}}{m_{b}}\right) \theta_{c}, \\
& V_{31} \simeq-\frac{1}{\sqrt{2}}\left(h_{u}+2 h_{d}\right) \theta_{c} \simeq\left(2 \frac{m_{s}}{m_{b}}-\frac{m_{c}}{m_{t}}\right) \theta_{c},
\end{aligned}
$$

and establish the $\theta_{c}$-independent relation

$$
\frac{V_{13}}{V_{31}} \simeq \frac{2 \frac{m_{c}}{m_{t}}-\frac{m_{s}}{m_{b}}}{2 \frac{m_{s}}{m_{b}}-\frac{m_{c}}{m_{t}}} .
$$

The relative minus signs in these equations are a nontrivial consequence of eq.(3.48).

Our final relation interrelates quark masses. From eq.(3.44) we learn that

$$
\frac{p_{d}}{p_{u}}=\left(\frac{c_{1} r_{u}^{2}+c_{2} r_{d}^{2}}{c_{1} r_{d}^{2}+c_{2} r_{u}^{2}}\right) .
$$

Our model does not tell us why $\left|h_{u}\right| \ll\left|h_{d}\right|$. It points, however, towards the parameter space region

$$
c_{1} \gg c_{2} .
$$

In this case,

$$
\frac{p_{d}}{p_{u}} \simeq\left(\frac{r_{u}}{r_{d}}\right)^{2}
$$

leading to

$$
\frac{h_{u}}{h_{d}} \simeq-\left(\frac{r_{u}}{r_{d}}\right)^{2 / 3}
$$

Furthermore, invoking the see-saw weak isospin restriction which implies $\lambda_{u}=\lambda_{d}$ and $\Lambda_{u}=\Lambda_{d}$, and recalling the eigenvalue spectrum, we arrive at

$$
\frac{r_{u}}{r_{d}} \simeq \frac{m_{u} / m_{c}}{m_{d} / m_{s}} .
$$

Combining eq.(3.58) and eq.(3.47), an unusual mass relation makes its appearance:

$$
\frac{m_{u}}{m_{d}} \simeq\left(\frac{m_{b}}{m_{t}}\right)^{3 / 2}\left(\frac{m_{c}}{m_{s}}\right)^{5 / 2} .
$$

It is remarkable that the light quark mass ratio gets fixed by the heavy quark mass ratios.

\section{DISCUSSION AND CONCLUSIONS}

The scheme developed in this paper provides, we believe, a non-trivial framework that may contribute to a resolution of the flavor problem. It is successful in several very important respects:
1. It seamlessly merges the universal see-saw mechanism, Left-Right symmetry and horizontal symmetry in a coherent gauge theoretic structure.

2. It establishes a connection between the experimentally observed (approximate) geometric mass hierarchy and the appealing horizontal symmetry group $S U(3)_{H}$.

3. Further, it establishes a connection between the geometric mass hierarchy and threefold family replication.

4. The symmetry breaking pattern is interpreted in geometric terms. The observed mass and mixing angle hierarchies reflect a simple horizontal structure.

5. The CKM matrix has the correct qualitative pattern. 6 . The model gives rise to phenomenologically successful mass-ratio (rather than square-mass-ratio) mixing relations, and fixes the $\frac{m_{u}}{m_{d}}$ mass ratio.

Using the heavy quark masses as input, we can probe our predictions numerically. Given the following $m_{q}\left(m_{Z}\right)$ values 12

$$
\begin{aligned}
& m_{s} \approx 93 \mathrm{MeV}, \\
& m_{c} \approx 677 \mathrm{MeV}, \\
& m_{b} \approx 3.0 \mathrm{GeV}, \\
& m_{t} \approx 181 \mathrm{GeV},
\end{aligned}
$$

and the Cabibbo angle $\theta_{c} \simeq 0.22$, we estimate the other CKM mixings to be

$$
\begin{aligned}
V_{c b} & \simeq-V_{t s} \simeq \frac{m_{s}}{m_{b}}+\frac{m_{c}}{m_{t}} \simeq 0.035, \\
V_{u b} & \simeq\left(2 \frac{m_{c}}{m_{t}}-\frac{m_{s}}{m_{b}}\right) \theta_{c} \simeq 0.005, \\
V_{t d} & \simeq\left(2 \frac{m_{s}}{m_{b}}-\frac{m_{c}}{m_{t}}\right) \theta_{c} \simeq 0.013 .
\end{aligned}
$$

These values should be compared with the experimental averages (only the first two of which have been measured) 0.037 and 0.004 , respectively.

Given the $m_{q}(1 \mathrm{GeV})$ values [12] of

$$
\begin{aligned}
& m_{s} \approx 175 \mathrm{MeV}, \\
& m_{c} \approx 1.51 \mathrm{GeV}, \\
& m_{b} \approx 7.18 \mathrm{GeV}, \\
& m_{t} \approx 475 \mathrm{GeV},
\end{aligned}
$$

we predict a light quark mass ratio of

$$
\frac{m_{u}}{m_{d}} \simeq\left(\frac{m_{b}}{m_{t}}\right)^{3 / 2}\left(\frac{m_{c}}{m_{s}}\right)^{5 / 2} \simeq 0.44 .
$$

This result is to be compared 13 with 0.55 calculated by Gasser-Leutwyler and Dominguez-deRafael, with 0.29 advertized by Donoghue-Holstein-Wyler, and the 0.4 value derived by Narison.

There are also several important open problems facing our model. Of immediate relevance to the discussion in this paper, it is clear that the symmetry breaking analysis is incomplete. The complexity of the Higgs systems 
needed to break attractive and powerful higher symmetries is a general model building problem. In our case, while the Higgs boson multiplet structure has some appealing features, the analysis of the general Higgs potential is bedeviled by a proliferation of parameters. In this regard, the remarkable phenomenological success of the partial analysis presented above is encouraging. However, we do not as yet know whether the required VEV pattern exists as an absolute minimum of the Higgs potential for a range of parameters. To rephrase the question, we can ask: What is the simplest Higgs system that can furnish the VEV pattern we need? Is the system discussed in this paper sufficient, or are other multiplets needed? In particular, does supersymmetry, with its strong constraints on the Higgs potential, have an important role to play? (The cubic nature of superpotentials suggests an intriguing connection between $S U(3)_{H}$ invariance and the number of Yukawa legs). It is important to know how robust are our mass and mixing angle relations, because the partial Higgs potential minimization procedure was central in the derivation of some of them.

The role of even higher symmetries is also an open problem. We have seen that see-saw weak-isospin group $S U(2)_{L}^{\prime} \otimes S U(2)_{R}^{\prime}$ is immediately suggested by the multiplet structure of the theory. If it exists, it also requires that different Higgs sextets couple to the charge $+2 / 3$ and $-1 / 3$ sectors, which we invoked for phenomenological reasons. There is a further suggestive extension to grand unified $S O(10) \otimes S O(10)^{\prime}$, consistent with the flavorchiral nature of $S U(3)_{H}$. If $S U(3)_{H}$ is truly a global symmetry, as suggested by its flavor-chirality and consequent anomalies, then its deeper origin is another open problem. Indeed, the essential use of the $\underline{3} \oplus \underline{6}^{*}$ representation of $S U(3)_{H}$ hints at a possible underlying composite model, for exactly the same reasons that Gell-MannNe'eman SU(3) suggested the existence of quarks. The simple group-theoretic result that

$$
\underline{3}^{*} \otimes \underline{3}^{*}=\underline{3} \oplus \underline{6}^{*}
$$

may imply that the multiplet structure we have used can be constructed from two-body bound states of preons in the $\underline{3}^{*}$ representation of $S U(3)_{H}$.

The model presented in this paper does not explain the hierarchy between the top and bottom quark masses. Recall that there is one unsuppressed eigenvalue $\ell$ per charge sector, which indeed matches the observed value of $m_{t}$ beautifully. However, the relatively low mass for the bottom quark can only be incorporated through a Yukawa coupling constant hierarchy

$$
\frac{\lambda_{d}}{\lambda_{u}}=\frac{m_{b}}{m_{t}} .
$$

The theory as it stands does not address the question of mass splitting between weak-isospin partners. Several possible approaches to this deep issue are apparent: Perhaps the mass parameter $t$ in the effective $5 \times 5$ mass matrix $\mathcal{M}_{L R}$ is significantly larger for the charge $-1 / 3$ sector than it is for the charge $+2 / 3$ sector. In that case, the would-be order $\ell$ eigenvalue becomes see-saw suppressed. Another perspective on the top-bottom mass splitting issue is afforded by the question: Does custodial $\mathrm{SU}(2)$ have a role to play? This accidental symmetry of the minimal standard model Higgs potential, if enforced, leads to mass degeneracy between weak-isospin partner fermions. Interestingly, custodial $\mathrm{SU}(2)$ can sometimes be identified with $S U(2)_{R}[14]$.

We have not explicitly discussed leptons in this paper, though the most straightforward extension of our model to leptons is obvious. The full neutral-lepton mass matrix is then an $18 \times 18$ matrix which should have interesting calculable features. In the simple universal see-saw model, the Majorana see-saw mechanism for neutrinos is automatic, leading to enhanced suppression for neutrino masses. A similar phenomenon is expected here, but the question of whether the light neutrinos are necessarily hierarchical needs to be answered. The mass splitting between quarks and leptons is another deep issue. One well-known approach to this problem lies in the exploration of symmetries between quarks and leptons, for example grand unification, Pati-Salam SU(4), and discrete quark-lepton symmetry 15.

Finally, there is the question of experimental testability. The phenomenology of the familon-like Goldstone bosons arising from the breakdown of global $S U(3)_{H}$ needs to be analyzed. Also, the production of cosmological texture in the early universe and its role in largescale structure formation may lead to a cosmological test of spontaneously broken global $S U(3)_{H}$. The mass and mixing angle relations we derived will be tested further as more data is gathered, particularly for the top-quark CKM elements $V_{32}$ and $V_{31}$. If right-handed weak interactions should be discovered, then our theory predicts that the up and down quarks will couple more strongly to $W_{R}$ and $Z_{R}$ than will charm, strange, top and bottom. Finally, the neutral-lepton mass matrix may have testable consequences for neutrinos, an issue we intend to explore in the future.

\section{ACKNOWLEDGMENTS}

AD would like to thank Professor Bruce McKellar and the School of Physics at The University of Melbourne for the enjoyable semester during which much of this research was carried out. RRV would like to thank the Physics Department at Ben-Gurion University for their kind hospitality during the completion of this work. RRV is supported by the Australian Research Council. 
[1] See, for example, H. Fritzsch, Nucl. Phys. B70, 436 (1977); R. Barbieri, R. Gatto and F. Strocchi, Phys. Lett. B74, 344 (1978); T. Maehara and T. Yanagida, Prog. Theor. Phys. 60, 822 (1978); C. Froggatt and H.B. Nielsen, Nucl. Phys. B147,277 (1979); F. Wilczek and A. Zee, Phys. Rev. Lett. 42, 421 (1979); A. Davidson, M. Koca and K.C. Wali, Phys. Rev. Lett. 43, 92 (1979); A. Davidson, M. Koca and K.C. Wali, Phys. Rev. D20, 1195 (1979); C.L. Ong, Phys. Rev. D19, 2738 (1979); J. Chakrabarti, Phys. Rev. D20, 2411 (1979); A. Davidson and K.C. Wali, Phys. Rev. D21, 787 (1980); C.L. Ong, Phys. Rev. D22, 2886 (1980); H. Harari and N. Seiberg, Phys. Lett. B102, 263 (1981); G. Ecker, Z. Phys. C24 353 (1984); A. Davidson, V.P. Nair and K.C. Wali, Phys. Rev. D29, 1504 (1984); T.K. Kuo and N. Nakagawa, Nucl. Phys. B250, 641 (1985); K.S. Babu and X.G. He, Phys. Rev. D36, 3484 (1987); R. Foot, G.C. Joshi, H. Lew and R.R. Volkas, Phys. Lett. B226, 318 (1989); K.S. Babu and R.N. Mohapatra, Phys. Rev. D43, 2278 (1991); K. Bandyopadhyay and D. Choudhury, Phys. Rev. D43, 1646 (1991); M.T. Yamawaki and W.W. Wada, Phys. Rev. D43, 2432 (1991); A.A. Bagneid, T.K. Kuo and G.T. Park, Phys. Rev. D44, 2188 (1991); D.S. Shaw and R.R. Volkas, Phys. Rev. D47, 241 (1992); G.T. Park and T.K. Kuo, Phys. Rev. D45, 1720 (1992); T.K. Kuo and G.T. Park, Phys. Rev. D50, 3508 (1994); L. Ibanez and G.G Ross, Phys. Lett. B322, 100 (1994); G. Anderson, S. Rabi, S. Dimopoulos, L.J. Hall and G.D. Starkman, Phys. Rev. D49, 3660 (1994); P.H. Frampton and T.W. Kephart, Int. J. Mod. Phys. A10, 4689 (1995); P. Binetray, S. Lavignac and P. Ramond, Nucl. Phys. B477, 353 (1996); P.H. Frampton and O.C.W. Kong, Phys. Rev. Lett. 77, 1699 (1996); 75, 781 (1995); Phys. Rev. D53, 2293 (1996); Z. Berezhiani, Nucl. Phys. (Proc. Suppl.) 52A,153 (1997); W.A. Ponce, L.A. Wills and A. Zepeda, Z. Phys. C73, 711 (1997).

[2] T. Yanagida, Phys. Rev. D20, 2986 (1979); G. Zoupanos, Phys. Lett. B115, 221 (1982); Y. Chikashige, G. Gelmini, R.D. Peccei and M. Roncadelli, Phys. Lett. B94, 499 (1980); E. Papantonopoulos and G. Zoupanos, Phys. Lett. B110, 465 (1982); Z.G. Berezhiani, Phys. Lett. 129, 99 (1983); G.B. Gelmini, J.M. G'erard, T. Yanagida and G. Zoupanos, Phys. Lett. 135, 103 (1984); K. Bandyopadhyay and A K. Ray, Phys. Rev. D38, 2231 (1988); Z.G. Berezhiani and M.Yu. Khlopov, Z. Phys. C49, 73 (1991); Z.G. Berezhiani and L. Lavoura, Phys. Rev. D45, 934 (1992); M. Joyce and N. Turok, Nucl. Phys. B416, 389 (1994).

[3] J.C. Pati and A. Salam, Phys. Rev. D8, 1240 (1973). For a recent attempt to use partial unification, see R. R. Volkas, Phys. Rev. D53, 2681 (1996).

[4] H. Georgi and S.L. Glashow, Phys. Rev. Lett. 32, 438 (1974). For some recent work on the fermion mass problem in grand unified models see, for instance, P. Ramond, R.G. Roberts and G.G. Ross, Nucl. Phys. B406, 19 (1993); S. Dimopoulos, L.J. Hall and S. Raby, Phys. Rev. D45, 4192 (1992).

[5] See, for example, B.S. Balakrishna, Phys. Rev. Lett. 60, 1602 (1988); B.S. Balakrishna, A.L. Kagan and R.N. Mohapatra, Phys. Lett. B205, 345 (1988); K.S. Babu and E.
Ma, Mod. Phys. Lett. A4, 1975 (1989); K.S. Babu, B.S. Balakrishna and R.N. Mohapatra, Phys. Lett. B237, 221 (1990); X.G. He, R.R. Volkas and D.D. Wu, Phys. Rev. D41, 1631 (1990); E. Ma, Phys. Rev. Lett. 64, 2866 (1990).

[6] A. Davidson and K.C. Wali, Phys. Rev. Lett. 59, 393 (1987); S. Rajpoot, Phys. Lett. B191, 122 (1987); A. Davidson, S. Ranfone and K.C. Wali, Phys. Rev. D41, 208 (1990); I. Sogami and T. Shinohara, Prog. Theo. Phys. 66, 1031 (1991); Z.G. Berezhiani and R. Rattazzi, Phys. Lett. B279, 124 (1992); A. Davidson, L. Michel, M.L. Sage and K.C. Wali, Phys. Rev. D49, 1378 (1994); P. Cho, Phys. Rev. D48, 5331 (1994); V. Jain and R. Shrock, Phys. Lett. B352, 83 (1995); W.A. Ponce, A. Zepeda and R.G. Lozano, Phys. Rev. D49, 4954 (1994); Y. Koide and H. Fusaoka, Z. Phys. C71, 459 (1996).

[7] M. Gell-Mann, P. Ramond and R. Slansky, in Supergravity, eds. P. van Nieuwenhuizen and D.Z. Freedman (North Holland, Amsterdam, 1979); T. Yanagida, in Proc. Unified Theory and Baryon Number in the Universe, ed. A. Sawada and H. Sugawara (KEK, Tsukuba-Gun, IbarakiKen, Japan, 1979).

[8] A. Davidson and K.C. Wali, Phys. Rev. Lett. 60, 1813 (1988); A. Davidson, Nucl.Phys. (Proc. Suppl.) B13, 555 (1990).

[9] See e.g. H. Haber and G. Kane, Phys. Rep. C117, 75 (1985); H.P. Nilles, Phys. Rep 110, 1 (1984); M. Leurer, Y. Nir and N. Seiberg, Nucl. Phys. B398, 319 (1993); D.J. Castano, E.J. Piad and P. Ramond, Phys. Rev. D49,4882 (1994); X. Tata, in QCD and Beyond hepph/9510287; J. Bagger, in Theor. Adv. Study Inst. (Boulder 1995) hep-ph/9604232; M. Drees hep-ph/9611409; H. Murayama and M. Peskin, Ann. Rev. Nucl. Part. Sci. 46, 533 (1996); S. Martin, in Perspectives in SUSY (ed. G. Kane) hep-ph/9709356; S. Dawson, Lecture notes (tasi97) hep-ph/9712464.

[10] See, for instance, F. Wilczek, Phys. Rev. Lett. 49, 1549 (1982); G.B. Gelmini, S. Nussinov and T. Yanagida, Nucl. Phys. B219, 31 (1983); D.A. Dicus and V.L. Teplitz, Phys. Rev. D28, 1778 (1983); A. Davidson and M.A.H. Vozmediano, Nucl. Phys. B248, 647 (1984) D.S.P. Dearborn, D.N. Schramm and G. Steigman, Phys. Rev. Lett. 56, 26 (1986).

[11] C. Barnes and N. Turok, Phys. Rev. D56, 1989 (1997).

[12] For a recent update, see e.g. H. Fusaoka and Y. Koide (hep-ph/9712201).

[13] J. Gasser and H. Leutwyler, Phys. Rep. 87, 77 (1982); C.A. Dominguez and E. deRafael, Ann. Phys. 174, 372 (1987); J.F. Donoghhue, B.R. Holstein and D. Wyler, Phys. Rev. Lett. 69, 3444 (1992); S. Narison, Phys. Lett. B358, 113 (1995); H. Leutwyler, Phys. Lett. B378, 313 (1996).

[14] See the paper by R.R. Volkas in Ref. [3].

[15] R. Foot and H. Lew, Phys. Rev. D41, 3502 (1990); R. Foot, H. Lew and R.R. Volkas, Phys. Rev. D44, 1531 (1991); R.R. Volkas, Phys. Rev. D50, 4628 (1994); R. Foot and R.R. Volkas, Phys. Lett. B358, 318 (1995) . 